\title{
Effects of diet and dietary experience on body weight regulation in rats with septal lesions
}

\author{
FREDERICK S. VOM SAAL, C. ROBIN TIMMONS, and LEONARD W. HAMILTON \\ Rutgers University, New Brunswick, New Jersey 08903
}

\begin{abstract}
A series of three experiments was conducted to assess whether septal lesions would affect the capacity of female rats to respond to changes in diet palatability. It was found that septal lesions produced overeating in rats shifted from an unpalatable powdered diet to a highly palatable mixture of Crisco and powdered food. However, overeating of the Crisco diet only occurred if septal-lesioned rats were first exposed to the powdered diet following surgery. Surprisingly, septal lesions did not affect responsivity to a shift from the Crisco to the powdered diet. The results indicate that, while septal damage appeared to alter responsivity to a change in diet, relative palatability factors alone are not the cause. Rather, septal lesions may produce permanent changes in the pattern of ingestive behavior when lesioned rats are exposed to certain diets following surgery.
\end{abstract}

There is considerable evidence which indicates that the septum is involved in modulating the rat's responsiveness to the taste and postingestive effects of food. A rat with septal damage increases its intake of a sucrose solution in response to taste rather than postingestive factors (Flaherty \& Hamilton, 1971; Hamilton, 1971) decreases its intake dramatically when the diet is adulterated with cellulose (Singh \& Meyer, 1968), undercompensates for stomach loading of nutrients (Hamilton, Capobianco, \& Worsham, 1974), and shows enhanced reactivity in a gustatory conditioned aversion paradigm (Hamilton \& Capobianco, 1973; McGowan, Garcia, Ervin, \& Schwartz, 1969).

Despite these findings, there are conflicting data concerning the role of the septum in the regulation of food intake and body weight. Although it has been reported that septal lesions produce hypophagia and a concommitant loss of body weight (Beatty \& Schwartzbaum, 1967), other investigators have reported no change in body weight and intake (Gurowitz \& Lubar, 1966; Stoller, 1972) or hyperphagia and increased body weight (Lorens \& Kondo, 1969; Singh \& Meyer, 1968) following septal lesions. Although the above differences may simply reflect damage to different sites within the septal complex, it seems reasonable to attribute changes in intake and body weight in lesioned rats at least in part to palatability effects, since a septal lesion produces hyperresponsivity to taste and olfactory cues (vom Saal, Hamilton, \& Gandelman, 1975). Therefore, the

This research was supported by grants from The Weight Watchers Foundation and The Rutgers Research Council. Reprint requests should be addressed to L. W. Hamilton, Psychology Department, Rutgers University, New Brunswick, New Jersey 08903. present experiments were designed to investigate the effects of septal lesions on the response to changes in diet palatability.

\section{EXPERIMENT 1}

The purpose of the first experiment was to demonstrate that the intake and body weight of a septal-lesioned rat can be determined by the presumed palatability of the diet. Female rats were employed since they are normally more responsive to taste factors than males, and it was anticipated that they would show greater changes in responsivity to taste cues following septal lesions. It was predicted that septal-lesioned rats maintained on the familiar, standard laboratory food pellets would show no change in food intake and rate of weight gain. However, when shifted to powdered laboratory chow, the lesioned rats were expected to decrease their intake and lose weight relative to controls, while a shift to a highly palatable, high-fat diet was expected to produce overeating and an increased rate of weight gain relative to the control rats.

\section{Methods}

Animals. Twelve female Sprague-Dawley albino rats, 200-225 g, were obtained from Carworth Farms (Wilmington, Mass.). All rats were isolated and allowed ad-lib access to both tap water and Purina Laboratory Chow (pellets). The colony was maintained at $22^{\circ} \mathrm{C}$ and kept on a $14 / 10 \mathrm{~h}$ light/dark cycle, with lights on at $0600 \mathrm{~h}$.

Procedures. After 7 days of adaptation to the laboratory environment, food and water intake and body weight measures were taken for 3 consecutive days. Based on these data, two matched groups of rats were formed. On the following day, designated Day 0 of the experiment, surgery was performed under Equithesin anesthesia (Jensen-Salsbury), with each rat receiving $0.25 \mathrm{cc} / 100 \mathrm{~g}$ body weight. Six rats received bilateral septal lesions 
using the following stereotaxic coordinates (De Groot, 1959): $\mathbf{A P}=+7.8 \mathrm{~mm} ; \mathrm{L}= \pm 0.8 \mathrm{~mm} ; \mathrm{H}=+1.2 \mathrm{~mm}$. The bilateral lesions were made using $1.5 \mathrm{~mA}$ of anodal current delivered through each electrode for $15 \mathrm{sec}$. Operated controls $(n=6)$ were treated similarly, except that after the dura was punctured, the electrode was not lowered into the brain.

For 26 days following surgery, all animals were maintained on the food pellet diet $(3.61 \mathrm{kcal} / \mathrm{g})$. Beginning on Day 27 of the experiment, all animals were allowed access to a diet of powdered Purina Laboratory Chow $(3.61 \mathrm{kcal} / \mathrm{g})$ for 11 days followed by an additional 11 days of access to a high-fat (Crisco) diet $(5.52 \mathrm{kcal} / \mathrm{g})$. The high-fat, high-palatability diet was composed of a mixture of 2 parts powdered food to 1 part solid Crisco shortening (Proctor and Gamble) by volume. Free access to water was allowed throughout all phases of the experiment. Food and water intake and body weight measures were recorded daily with corrections made for spillage.

Upon completion of testing, animals with septal lesions were given an overdose of Equithesin and perfused intracardially with physiological saline followed by a $10 \%$ formal saline solution. The brains were removed and stored in formal saline until frozen for sectioning. Every fifth $30-\mu$ section through the lesion site was saved and stained with cresyl violet to allow microscopic verification of the damage. Except when otherwise indicated, analyses of variance were used to analyze the data, with all significant measures having a probability value of $p<.01$.

\section{Results}

Histology. Each of the six experimental animals was found to have a large bilateral lesion of the septal area. Figure 1 depicts the maximum and minimum extent of the lesions.

Pellet diet. Figure 2 presents the mean daily intake of food (kcal) and water $(\mathrm{ml})$ as well as the average daily body weight gain for each group. As expected, analyses of variance failed to find any differences between the control and lesioned animals on any of these measures. Although water intake and body weights of the septal rats tended to be above control levels, the differences were not statistically significant.

Powdered diet. Although the rats with septal lesions appeared to consume more powdered food

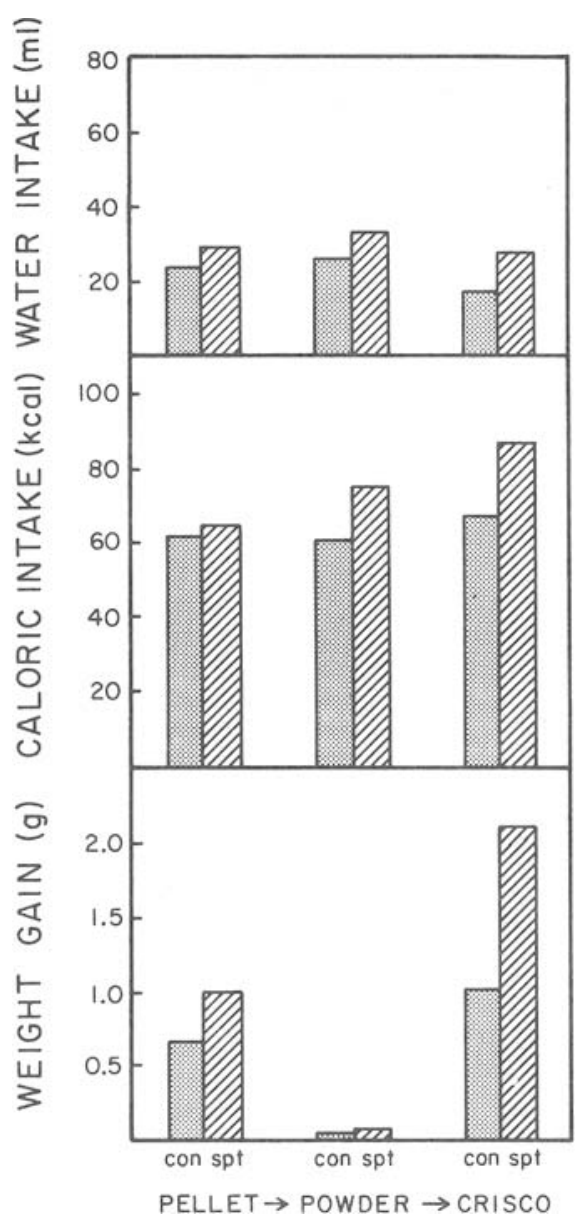

Figure 2. Mean daily weight gain and food and water intake for the animals in Experiment 1 while consuming the three different diets: pellet $=$ Purina Lab Chow; powder $=$ Purina powdered Lab Chow; Crisco $=$ a mixture of 2 parts powdered Lab Chow and 1 part solid Crisco shortening (by volume). Con = control rats; Spt $=$ septal-lesioned rats.
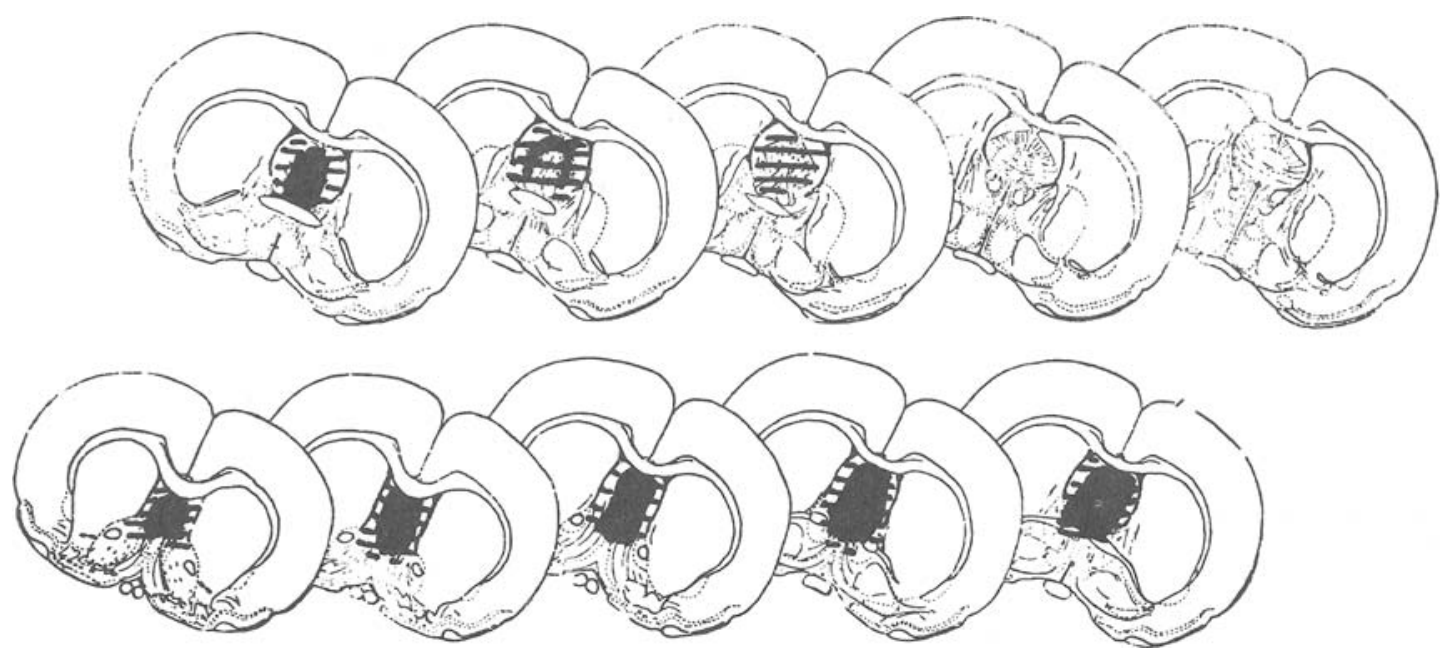

Figure 1. Reconstructions of the minimum (shaded) and maximum (lined) extent of brain damage. 
than the controls $[F(1,10)=6.88, p<.05]$, this difference was probably artificial. It was noted that upon being shifted to the powdered diet, four of the six lesioned animals spilled large quantities of the food powder, which made accurate determination of actual intake difficult. The fact that the septal and control rats gained almost no weight while consuming the powdered diet supports the conclusion that the apparent difference in food intake was probably due to the excess spillage on the part of the septal rats. There were no significant differences between the groups in the amount of water consumed each day.

Crisco diet. When the animals were shifted from the powdered food to the Crisco diet, the rats with septal lesions ceased spilling the food and consumed significantly more of the Crisco diet than the control rats $[F(1,10)=11.9]$. Analyses of the body weight data indicated that, while both the septal and control rats increased their rate of weight gain on the Crisco diet, the septal rats gained twice as much weight per day as the controls, resulting in a significant Lesion by Days interaction $[F(10,100)=4.63]$. As was the case with the other two diets, the rats with lesions consumed slightly more water than the control rats, but these differences did not reach statistical significance.

\section{EXPERIMENT 2}

Experiment 1 demonstrated that septal-lesioned rats will overeat when shifted from a powdered laboratory chow to a high fat diet. However, several alternative explanations of this phenomenon are possible. One possibility is that the overresponse to the Crisco diet depends upon relative taste factors, with the increased intake being a function of the prior postoperative exposure to the powdered food. Alternatively, the Crisco diet could be more palatable than the powdered diet in the absolute sense, with presentation of the Crisco diet immediately postsurgically producing hyperphagia similar to that observed when the diet was shifted from powder to Crisco. If this were, in fact, true, then it could be proposed that a septal lesion was altering body weight regulation in response to high-fat diets as a consequence of some metabolic change, rather than due to the high-fat diet's being more palatable than the previous diet of powdered chow.

It was predicted that septal rats would not differ from controls in the rate of weight gain when exposed to the Crisco diet immediately following surgery and only become hyperphagic and obese when shifted from the powdered to the Crisco diet. Such a finding would support the idea that it is the relative rather than absolute taste factors which are important and suggest that the observed overeating is not due to a primary change in body weight regulation. To test these alternatives, sham-operated rats and rats with septal lesions were given either the powdered food or the Crisco diet immediately following surgery and subsequently shifted to the alternate diet.

\section{Methods}

Animals. Forty-eight female albino rats of the Sprague-Dawley strain, weighing 200-225 g, were obtained from the Blue Spruce Farms (Altamont, New York) and maintained as in Experiment 1.

Procedures. Five days prior to the start of the experiment, all animals were given ad-lib access to Purina powdered Laboratory Chow. On Day 0 of the experiment, 24 rats received bilateral septal lesions, following the procedure described in Experiment 1, and 24 rats served as operated controls. Assignment to surgical groups depended upon the intake and body weight measures recorded during the last 3 days of access to the powdered food.

Upon being returned to their home cages, the animals were further subdivided into four groups. Twelve septal lesioned $($ Spt $\mathrm{P} / \mathrm{C})$ and 12 control animals $($ Con $\mathrm{P} / \mathrm{C})$ continued to receive the powdered food diet for 27 days before being shifted to the Crisco diet for an additional 15 days. The remaining 24 animals, 12 septals (Spt C/P) and 12 controls (Con C/P), were maintained on the Crisco diet for the first 27 days postoperatively and then switched back to powdered food for the last 15 days. Water was available ad lib throughout the experiment. Food and water intake and body weight measures were recorded daily. To facilitate collection of spilled food, a fine mesh screen was placed under each cage. At the end of the experiment, the lesioned animals were sacrificed to allow histological examination as described in Experiment 1.

\section{Results}

Histology. Eight animals in Group Spt $\mathrm{P} / \mathrm{C}$ and 10 animals in Group Spt C/P were judged to have large bilateral lesions of the septum similar to those shown in Figure 1. The data for the other 6 lesioned animals were not included in the analyses. One animal in each of the control groups died prior to the end of the experiment, so these data also were discarded.

Preshift phase. Neither septal group differed from controls given the same diet in the rate of weight gain during the preshift phase of the experiment (see Figure 3). However, the animals given the Crisco diet gained more weight than similarly operated animals maintained on the powdered food, as indicated by a Diet by Days interaction for the lesioned animals $[\mathrm{F}(8,129)=8.65]$ and for the control animals $[F(8,161)=16.05]$. There was no differences in caloric intake between any of the groups during the preshift phase of the experiment. However, observation of the rats given the powdered food revealed that they spilled considerably more food than the rats given the Crisco diet, which may have influenced the food intake measurements.

Although the control animals given the powdered food during the preshift phase of the experiment (Con $\mathrm{P} / \mathrm{C})$ consumed more water per day than the control group maintained on the Crisco diet 


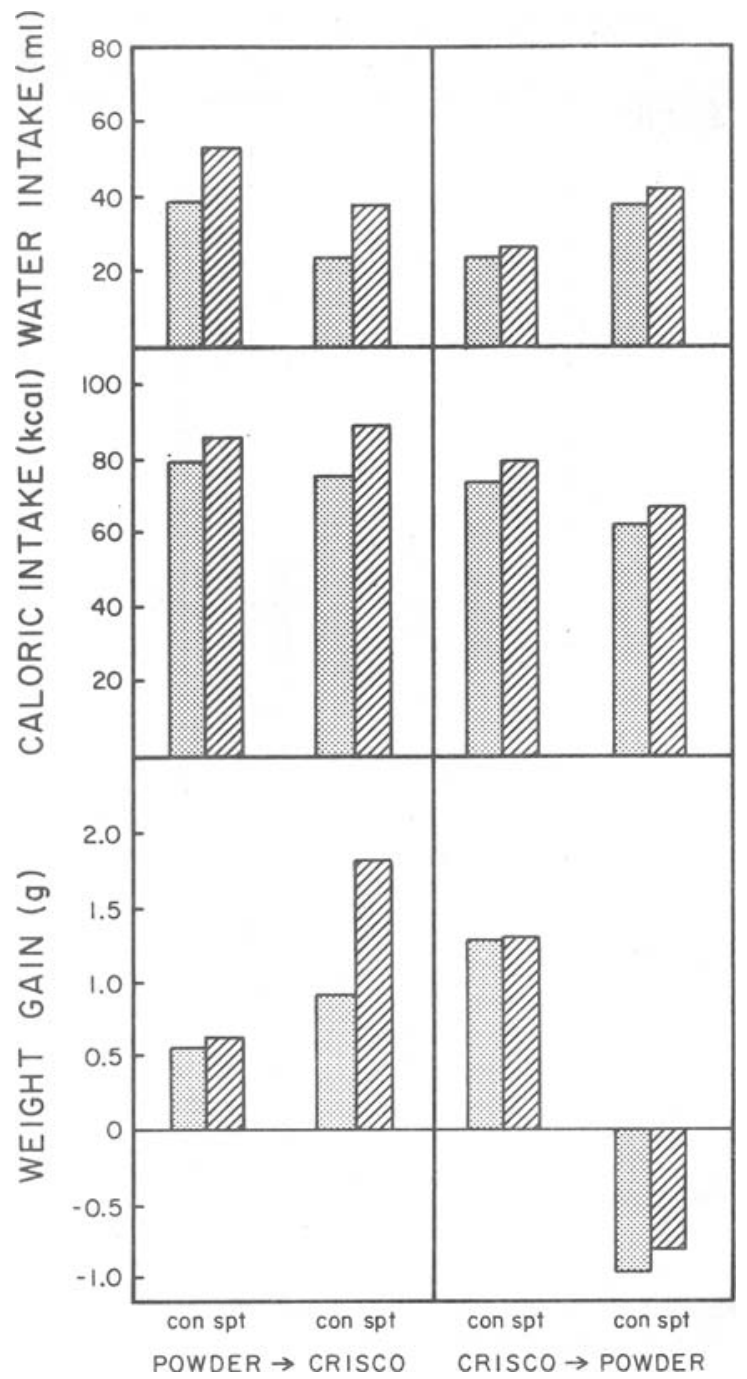

Figure 3. Mean daily weight gain and food and water intake for the animals in Experiment 2 during both the preshift and postshift phases of the experiment. Con $=$ control rats; Spt $=$ septal-lesioned rats.

$($ Con $\mathrm{C} / \mathrm{P})[\mathrm{F}(1,20)=24.99]$, the $\mathrm{ml}$ water $/ \mathrm{g}$ food ratio was the same for both groups. The rats with septal lesions given powdered food (Spt P/C) consumed more water than the powder control group (Con $\mathrm{P} / \mathrm{C})[\mathrm{F}(1,17)=9.34]$ and also consumed more water than the lesioned animals (Spt C/P) given the Crisco diet $[\mathrm{F}(1,16)=42.5]$. In addition, the $\mathrm{ml}$ water/g food ratio was significantly higher for the rats in Group Spt $\mathrm{P} / \mathrm{C}$ than for the rats in Group Con $\mathrm{P} / \mathrm{C}[\mathrm{F}(1,17)=6.3, \mathrm{p}<.05]$.

Postshift. Group Spt P/C consumed more of the Crisco diet $[\mathrm{F}(1,17)=6.88]$ and gained twice as much weight $[F(4,68)=4.25]$ as the controls (Con $\mathrm{P} / \mathrm{C}$ ) during the postshift phase. A comparison of rate of weight gain indicated that the rats with septal lesions (Spt $\mathrm{P} / \mathrm{C}$ ) gained weight faster during postshift consumption of the Crisco diet than lesioned rats (Spt $\mathrm{C} / \mathrm{P}$ ) consuming the Crisco diet during the preshift phase of the experiment $[F(4,64)$ $=3.73]$. The water intake of the rats in Group Spt P/C dropped when shifted from the powdered food to the Crisco diet, but not to the level of the preshift water intake of Group Spt $\mathrm{C} / \mathrm{P}[\mathrm{F}(1,16)=$ 11.0]. The water intake for Group Con $P / C$ dropped to the level exhibited by the rats in Group Con $\mathrm{C} / \mathrm{P}$ while consuming the Crisco diet during the preshift phase. This resulted in the rats in Group Spt $\mathrm{P} / \mathrm{C}$ continuing to consume $20 \mathrm{ml} /$ day more water than the rats in Group Con $\mathrm{P} / \mathrm{C}[\mathrm{F}(1,17)=15.9]$. The $\mathrm{ml}$ water/g food ratio for both Groups Spt P/C and Group Con $\mathrm{P} / \mathrm{C}$ did not change from preshift levels during the postshift phase, with the result that the $\mathrm{ml}$ water/g food ratio for Group Spt P/C remained significantly elevated above Group Con $\mathrm{P} / \mathrm{C}$ levels $[F(1,17)=8.11, p<.05]$.

Rats in Group Spt C/P did not differ from Group Con $\mathrm{C} / \mathrm{P}$ in their response to the shift to powdered food. Both groups lost weight over the first 10 days of the postshift phase and then gradually began to gain weight again, resulting in a significant Days effect $[F(4,76)=18.0]$. Thus, the rats with septal lesions overresponded relative to the controls to the shift from the powdered to the Crisco diet but not to the shift from the Crisco to powdered diet. The rats in Group Spt C/P and Con C/P did not differ from each other in food or water intake, but both groups gradually increased their intake of food and water over the 15 postshift days (after a large initial drop in food intake), as indicated by a significant Days effect for both food $[F(4,76)=18.0]$ and water $[F(4,76)=13.3]$. The increase in water and food intake for these animals paralled each other, since the $\mathrm{ml}$ water/g food ratio remained constant throughout the postshift phase for both groups.

\section{EXPERIMENT 3}

The results of Experiments 1 and 2 indicate that in septal-lesioned rats, food and water intake, and consequently body weight changes, following a shift from one diet to another depend upon prior dietary experience. Surprisingly, in Experiment 2, in which the rats were exposed to the powdered food for 5 days prior to surgery, the septal rats did not differ from controls in their rate of weight gain while on the powdered diet either during the preshift or postshift phase.

A third experiment attempted to determine the effects of prolonged exposure to the powdered food diet prior to surgery on the response of septal rats to a shift from the powdered to the Crisco diet following surgery. Additionally, since the prior studies had forced the animals to shift from one diet to another, this study allowed a choice between the 
two diets during the postshift phase of the study. Animals with septal lesions have been described as "perseverative." Therefore, it was thought that the choice situation would reveal any perseverative dietary responses which were precluded by the design of the previous experiments.

\section{Methods}

Animals. Fourteen female first-generation descendents of Sprague-Dawley albino breeding stock obtained from Blue Spruce Farms (Altamont, New York) were used in this experiment. Six weeks prior to the start of the experiment, the rats were housed individually and maintained as in the previous experiments, except that they received powdered rather than pelleted food. At the time of surgery, the animals weighed between 200 and $230 \mathrm{~g}$.

Procedures. For 6 weeks prior to surgery, the rats were allowed ad-lib access to Purina powdered Laboratory Chow and water. Assignment to surgical groups depended upon powdered food consumption, water intake, and body weight baseline data collected for 12 days prior to surgery. On Day 0 of the experiment, eight rats received septal lesions and six rats received control operations as described in Experiment 1. Following surgery, all rats received 42 days of ad-lib access to Purina powdered Laboratory Chow and water. During the last 16 of the 42 days, the rats were also given ad-lib access to the Crisco diet described in Experiment 1. The preshift phase thus lasted 27 days, and the postshift choice phase 16 days. Food and water intake and body weight measures were recorded daily with corrections made for spillage. Histological procedures followed those outlined in Experiment 1.

\section{Results}

Histology. All eight lesioned animals were found to have large lesions of the septal area similar to the lesions depicted in Figure 1.

Preshift. Following surgery, the rats with septal lesions consumed a significantly greater amount of the powdered food than the controls (see Figure 4). The largest increase in the rate of caloric intake occurred during the first 10 days following surgery which then leveled off during the last 16 days of the preshift phase, yielding a significant Lesion by Days interaction $[\mathrm{F}(11,132)=6.12]$. However, during the last 16 preshift days, the lesioned rats still consumed more calories than the control rats $[F(1,12)=5.90]$, with both groups increasing their intake over days $[F(7,84)=3.22]$. Only two of the lesioned animals were observed to spill the powdered food more than the control animals.

Analyses of body weight data revealed similar patterns. Following surgery, the rats with septal lesions showed a transient increase in rate of body weight gain and then returned to parallel control levels, yielding a significant Lesion by Days interaction $[F(12,144)=3.90]$. During the last 16 days of the preshift phase, the lesioned animals maintained a higher body weight than the control animals $[F(1,12)$ $=55.26]$, although the lesioned animals gained weight at the same rate as the controls.

During the last 16 days of the preshift phase, the lesioned rats consumed a greater amount of water

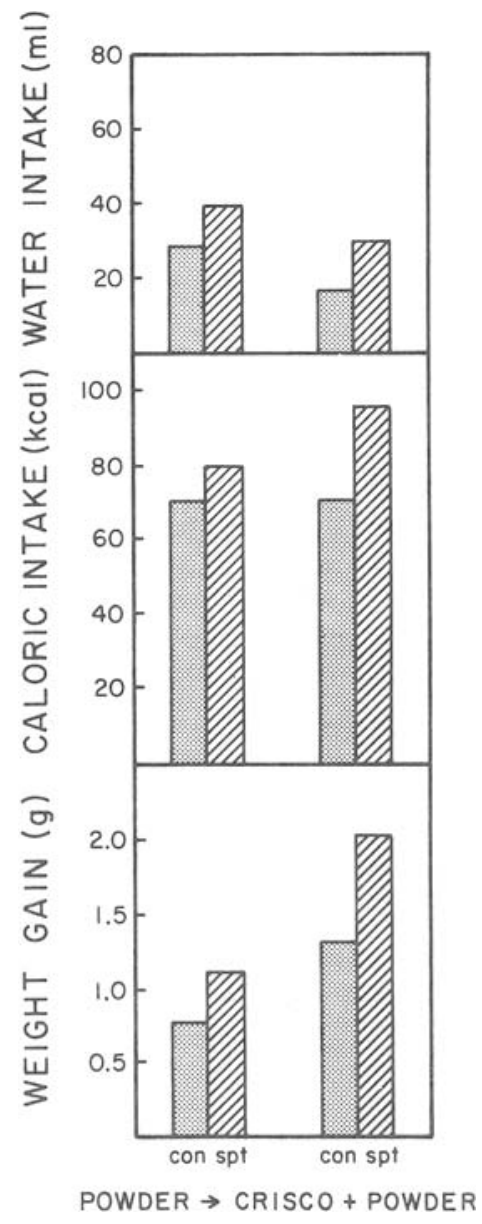

Figure 4. Mean daily weight gain and food and water intake for the animals in Experiment 3 during both the preshift and postshift phases of the experiment. Crisco + powder $=a$ choice of either powdered Lab Chow diet (powder) or the Crisco diet $($ Crisco $)$. Con $=$ control rats; Spt $=$ septal-lesioned rats.

$[\mathrm{F}(1,12)=24.77]$ and maintained a higher $\mathrm{ml}$ water/g food ratio $[F(1,12)=11.67]$ than the control rats.

Postshift. When given the choice of consuming either the Crisco or powdered diets, both groups decreased their intake of the powdered food to $10 \mathrm{kcal} /$ day. The animals with septal lesions consumed a significantly greater amount of the Crisco diet than the controls $[F(1,12)=6.82]$, with both groups increasing their intake of the diet over days $[F(7,84)=4.31]$. However, since the rats with septal lesions consumed more calories per day than the controls during the preshift phase of the experiment, another analysis compensating for this preshift difference was performed. An estimate of the expected caloric intake per day during the postshift phase was obtained from a regression equation based upon the caloric intake data from the preshift phase of the experiment. An analysis of the difference be- 
tween the actual caloric intake and that predicted by an extension of preshift levels of intake indicated that both groups showed significantly increased caloric intake over days $[F(7,84)=7.26]$; this increase was larger for septal-lesioned rats than for controls $[F(1,12)=6.0]$. Spillage was minimal for all animals during the postshift phase of the experiment.

Since the preshift body weight data also indicated that the lesioned animals were significantly different from the controls, the same rationale was used to analyze the difference between each animal's actual body weight and its predicted body weight. Although both groups showed a significant weight gain during the postshift period, a Lesion by Days interaction $[F(7,84)=2.20]$ indicated that when shifted to the Crisco diet, the animals with septal lesions gained weight at a faster rate than controls, even when corrected for the higher rate of weight gain that existed prior to the shift in diet.

Although the rats with septal lesions decreased their water intakes during the postshift phase $[F(1,112)=63.1]$, as did the control rats $[F(1,80)$ $=234.1$, lesioned animals still consumed significantly more water than the controls $[F(1,12)=$ 21.11]. The $\mathrm{ml}$ water/g food ratio did not change from the preshift levels for either the rats with the septal lesions or the controls during the postshift phase. However, during both the preshift and postshift phases, the $\mathrm{ml}$ water/g food ratio was higher for the septal-lesioned animals than for the controls.

\section{DISCUSSION}

The present experiments demonstrate that the effect of septal lesions on intake and body weight depends upon dietary factors as well as prior dietary experience. Given a mixture of powdered Lab Chow and Crisco shortening, rats with septal lesions consumed more of the Crisco diet and gained more weight than controls only when they had first had prior experience with the powdered Lab Chow alone following surgery. Exposure to the Crisco diet immediately postsurgically did not produce this response.

The simplest explanations of overeating the Crisco diet by septal rats would be to propose either a metabolic deficit or an impaired ability to monitor postingestive feedback. However, the observation that this overeating depends on prior experience strongly suggests that the explanation for the effect can be attributed to behavioral rather than metabolic dysfunctions. The question is, what type of behavioral response can account for the pattern of results obtained in this study?

A possible mechanism for the overeating of the Crisco diet is an exaggerated response to the relative taste factors following septal damage. Positive con- trast effects have been reliably demonstrated in a variety of situations (cf. Flaherty \& Largen, 1975), and rats with septal lesions are known to be overresponsive to both absolute and relative taste factors (e.g., Flaherty \& Hamilton, 1971). Despite the face validity, our acceptance of this explanation was tempered by several observations in this and other studies. The most disturbing observation was the failure of the rats with septal lesions to show a more exaggerated negative response than the controls to the shift from the Crisco diet to the less palatable powdered Lab Chow in Experiment 2. An exaggerated negative response did occur to the Crisco/ powder shift in Experiment 2 relative to the response to the pellet/powder shift in Experiment 1. But, in neither case did the septal-lesioned and control rats differ. Specifically, both control and septal-lesioned rats stopped gaining weight, but did not lose weight, when shifted from the pellet to the powdered diet in Experiment 1. A much more pronounced response to the Crisco/powder shift was observed in Experiment 2. Although not differing, the septal-lesioned and control rats lost an average of $15 \mathrm{~g}$ within 10-12 days following the shift from Crisco to powder before they finally began gaining weight again.

Although it cannot definitely be ruled out that a more exaggerated negative response to the shift to the powdered diet by the septal-lesioned rats relative to controls would have been found in Experiment 2 had the rats not been exposed to the powdered diet for 5 days prior to surgery, this is considered to be unlikely. A more exaggerated positive response to the shift from the powdered to the Crisco diet by the septal-lesioned rats occurred in all three experiments. Since the duration of preoperative exposure to the powdered diet varied across all three experiments (Experiment 1, 0 days; Experiment 2, 5 days; Experiment 3, 6 weeks), the exaggerated response of septal rats to the shift from the powdered to Crisco diet appears to be independent of preoperative exposure to the powdered food. The most convincing evidence that rats with septal lesions overrespond to taste factors has been based on experiments involving negative taste factors (e.g., Donovick, Burright, \& Zuromski, 1970). Furthermore, contrast effects in general tend to be somewhat transient, and the available data suggest that the effect may be even more transient in septal than in normal rats (unpublished observations). In conclusion, then, it may be premature to eliminate relative taste factors as an explanation of the overresponse to the Crisco diet, but there are enough conflicting findings to cast considerable doubt on this explanation.

An alternative explanation is that the combination 
of septal lesions and certain dietary experiences can lead to long-term, perhaps even permanent, changes in the pattern of ingestive behavior. Indeed, one of the first indications that the septum is involved in ingestive behavior showed just such a pattern: In 1965, Harvey and Hunt showed that postoperative water deprivation experience enhanced the tendency of rats with septal lesions to consume more water, and that this effect continued long after the lesioned animals were returned to free access to water. Similarly, in both Experiment 2 and Experiment 3, it was found that septal-lesioned rats consumed about $15-20 \mathrm{ml}$ more water per day than controls when given the powdered diet following surgery, and that this relative difference persisted when shifted to the Crisco diet during the postshift phase. When given the Crisco diet following surgery, however, septal and control rats did not differ from each other in their water consumption while on the Crisco diet or subsequently when shifted to the powdered diet. Thus, the pattern of water intake established by the septal rats subsequent to surgery appears to be permanent in that, once established, it is independent of dietary changes. In addition, in Experiment 1, the absence of a significant increase in water intake relative to controls for the septallesioned rats may have been due to the intake pattern established while consuming the pellet diet following surgery. It is possible that the pellet diet, which is not as dry and unpalatable as the powdered diet, does not result in the establishment of high levels of water intake as readily as the powdered diet does.

It is also interesting to note that in all three experiments the means for the septal-lesioned rats on all measures were consistently, although not always significantly, higher than the means for control animals. This result could indicate a tendency for rats to increase intake and body weight following septal lesions, but that this tendency can be modified by the sequence of diets presented both before and subsequent to surgery. For instance, the septal-lesioned rats gained significantly more weight than controls while consuming the powdered diet immediately following surgery in Experiment 3, but not in Experiment 2 (Groups $\mathrm{P} / \mathrm{C}$ ). This finding suggests that the 6 weeks of exposure to powdered food prior to surgery in Experiment 3 significantly altered the response to this diet immediately following damage to the septum relative to the effect that 5 days of preoperative exposure to powdered food had in Experiment 2.

At the time the present experiments were concluded, there was no direct evidence for major differences in feeding patterns of rats with septal lesions (Dickinson, 1973). Recent experiments in this laboratory (Sunday, Hamilton \& Timmons, unpublished observations) now indicate that septal lesions can produce a dramatic shift in feeding patterns. Following surgery, septal-lesioned rats maintained on powdered food develop a pattern of almost continuous consumption during the dark period of the light/ dark cycle. This contrasts sharply with the pattern of consuming discrete meals during the dark phase at about 90 -min intervals by normal rats maintained on a powdered diet. It is possible that the meal pattern established by septal-lesioned rats during the postoperative period may be related to the dry, relatively unpalatable characteristics of the powdered diet, and that this pattern of feeding may persist when the rats are later shifted to the Crisco diet. Implicit in this notion is the prediction that rats with septal lesions would not show a similar shift in the pattern of feeding when given the Crisco diet immediately postoperatively, a hypothesis that is yet to be tested.

Although further experiments will be necessary to test specific aspects of these hypotheses, the present data are clear in demonstrating the important effects of dietary history. The consummatory behavior of rats with septal lesions is not a simple function of the immediate taste and postingestive factors, but, rather, depends on a constellation of factors. Given these observations, it seems likely that the explanation of many of the effects of damage to the septum (and other limbic system structures) may ultimately be shown to be related to more general aspects of behavioral control rather than to interference with specific aspects of feeding circuits.

\section{REFERENCES}

Beatty, W. W., \& Schwartzbaum, J. S. Enhanced reactivity to quinine and saccharine solutions following septal lesions in the rat. Psychonomic Science, 1967, 8, 483-484.

DEGROOT, J. The rat forebrain in stereotaxic coordinates. Amsterdam: N.V. Noorde-Hollansche Uitgevers Maatschappij, 1959.

Dickinson, A. Prandial drinking following septal lesions in rats. Physiology \& Behavior, 1973, 10, 335-338.

Donovick, P. J., BurRight, R., \& ZUROMSKI, E. Localization of quinine aversion within the septum, habenula and interpeduncular nucleus of the rat. Journal of Comparative and Physiological Psychology, 1970, 71, 376-383.

Flaherty, C. F., \& Hamilton, L. W. Responsivity to decreasing concentrations of sucrose following septal lesions in the rat. Physiology \& Behavior, 1971, 6, 431-437.

Flaherty, C. F., \& Largen, J. Within subjects positive and negative contrast effects in rats. Journal of Comparative and Physiological Psychology, 1975, 88, 653-664.

Gurowitz, E. M., \& Lubar, J. P. Changes in activity, food ingestion, and passive avoidance behavior following limbic-septal ablation in the cat. Proceedings of the 74th Annual Convention of the American Psychological Association, 1966, 1, 107-108.

Hamilton, L. W. Starvation induced by sucrose ingestion in the rat: Partial protection by septal lesions. Journal of Comparative and Physiological Psychology, 1971, 77, 59-69.

Hamilton, L. W., \& Capobianco, S. Consumption of sodium chloride and lithium chloride in normal rats and rats with septal lesions. Physiological Psychology, 1973, 1, 213-218. 
Hamilton, L. W., Capobianco, S., \& Worsham, E. Lowered response to postingestive cues following septal lesions in rats. Journal of Comparative and Physiological Psychology, 1974, 87, 131-141.

HaRVey, J., \& Hunt, H. Effect of septal lesions on thirst in the rat as indicated by water consumption and operant responding for water reward. Journal of Comparative and Physiological Psychology, 1965, 59, 49-56.

LoRENs, S. A., \& Kondo, C. Y. Effects of septal lesions on food and water intake and operant responding for food. Physiology \& Behavior, 1969, 4, 729-732.

McGowan, B. K., Garcia, J., Ervin, F. R., \& Schwartz, J. Effects of septal lesions on bait-shyness in the rat. Physiology \& Behavior, 1969, 4, 907-909.
SINGH, D., \& MEYER, D. Eating and drinking by rats with lesions of the septum and the ventromedial hypothalamus. Journal of Comparative and Physiological Psychology, 1968, 65, 163-166.

StolleR, W. L. Effects of septal and amygdaloid lesions on discrimination, eating, and drinking. Physiology \& Behavior, 1972, 8, 823-828.

vom SaAl, F. S., Hamiton, L. W., \& Gandelman, R. J. Faster acquisition of an olfactory discrimination following septal lesions in male albino rats. Physiology \& Behavior, 1975, 14, 697-703.

(Received for publication December 21, 1977; revision accepted April 3, 1978.) 